AEI-2010-124

\title{
Towards Field Theory Amplitudes From the Cohomology of Pure Spinor Superspace
}

\author{
Carlos R. Mafral \\ Max-Planck-Institut für Gravitationsphysik, Albert-Einstein-Institut \\ 14476 Golm, Germany
}

\begin{abstract}
A simple BRST-closed expression for the color-ordered super-Yang-Mills 5-point amplitude at tree-level is proposed in pure spinor superspace and shown to be BRSTequivalent to the field theory limit of the open superstring 5-pt amplitude. It is manifestly cyclic invariant and each one of its five terms can be associated to the five Feynman diagrams which use only cubic vertices. Its form also suggests an empirical method to find superspace expressions in the cohomology of the pure spinor BRST operator for higherpoint amplitudes based on their kinematic pole structure. Using this method, Ansätze for the 6- and 7-point 10D super-Yang-Mills amplitudes which map to their 14 and 42 color-ordered diagrams are conjectured and their 6- and 7-gluon expansions are explicitly computed.
\end{abstract}

July 2010

1 email: crmafra@aei.mpg.de 


\section{Introduction}

As Parke and Taylor have shown for MHV amplitudes [1], it is sometimes possible to obtain simple expressions for seemingly complicated Yang-Mills amplitudes in four spacetime dimensions. Using the pure spinor formalism [2] and its pure spinor superspace [3] (see also [4]) it will be proved that the tree-level color-ordered five-point super-Yang-Mills amplitude in ten dimensions can be written simply as

$$
\mathcal{A}_{5}(1,2,3,4,5)=\frac{\left\langle L_{45} L_{12} V^{3}\right\rangle}{s_{45} s_{12}}+\operatorname{cyclic}(12345),
$$

where $V^{j}$ is the unintegrated massless vertex operator and $L_{i j}$ is related to the OPE of a unintegrated and an integrated vertex operator in a way to be defined below.

It will also be suggested that higher-point amplitudes might have simple forms like the above, as there seems to be a direct correspondence between superspace expressions and Feynman diagrams which use only cubic vertices as in the arguments of [5]. Using the empirical method described in subsection 3.1, it will be argued that the super-Yang-Mills 6- and 7-point color-ordered amplitudes are proportional to

$$
\begin{gathered}
\mathcal{A}_{6}(1,2,3,4,5,6)=\frac{\left\langle L_{12} L_{34} L_{56}\right\rangle}{3 s_{1} s_{3} s_{5}} \\
+\frac{1}{2} \frac{\left\langle T_{123}\right.}{s_{1} t_{1}} \frac{\left(V^{4} L_{56}\right.}{s_{5}}+\frac{\left.\left.L_{45} V^{6}\right)\right\rangle}{s_{4}}-\frac{1}{2} \frac{\left\langle T_{126}\right.}{s_{1} t_{3}} \frac{\left(V^{3} L_{45}\right.}{s_{4}}+\frac{\left.\left.L_{34} V^{5}\right)\right\rangle}{s_{3}}+\operatorname{cyclic}(1 \ldots 6)
\end{gathered}
$$

and

$$
\begin{gathered}
\mathcal{A}_{7}(1,2,3,4,5,6,7)=\frac{\left\langle T_{231} L_{45} L_{67}\right\rangle}{s_{2} t_{1} s_{4} s_{6}}+\frac{\left\langle T_{123} T_{564} V_{7}\right\rangle}{s_{1} t_{1} s_{5} t_{4}}+\frac{\left\langle T_{127} T_{345} V_{6}\right\rangle}{s_{1} t_{7} s_{3} t_{3}} \\
-\frac{\left\langle T_{123} T_{456} V_{7}\right\rangle}{s_{1} t_{1} s_{4} t_{4}}-\frac{\left\langle T_{127} T_{453} V_{6}\right\rangle}{s_{1} t_{7} s_{4} t_{3}}-\frac{\left\langle T_{123} L_{45} L_{67}\right\rangle}{s_{1} t_{1} s_{4} s_{6}}+\operatorname{cyclic}(1 \ldots 7)
\end{gathered}
$$

where $T_{i j k}$ is related to the OPE of one unintegrated and two integrated vertices in a way to be defined below and $s_{1}, \ldots, s_{6}$ and $t_{1}, \ldots, t_{3}\left(s_{1}, \ldots, s_{7}\right.$ and $\left.t_{1}, \ldots, t_{7}\right)$ are the 6 -point (7-point) generalized Mandelstam variables of [6,7]. Using a computer program [8], the 6and 7-gluon expansions of (1.2) and (1.3) are computed in Appendix $\mathrm{B}$ 目.

2 In the amplitude computations of [6, ]] the results were written in the $4 \mathrm{D}$ helicity formalism language, so a $10 \mathrm{D}$ comparison of results is not straightforward. However a comparison to the result [9] should be made [10]. After the first version of this paper came out, the 6-gluon amplitude has been successfully matched against the results of Zvi Bern, which he kindly provided [11]. The 7-gluon amplitude still remains to be checked. 
Furthermore, given that the tree-level SYM 4-point amplitude can be written as [12]

$$
\mathcal{A}_{4}(1,2,3,4)=\frac{1}{s_{12}}\left\langle L_{12} V^{3} V^{4}\right\rangle+\frac{1}{s_{41}}\left\langle L_{41} V^{2} V^{3}\right\rangle,
$$

it is pointed out that the four-point Jacobi-like Bern-Carrasco-Johansson kinematic identity [5] becomes

$$
\left\langle L_{\{12} V_{3\}} V_{4}\right\rangle=0,
$$

where $\{i j k\}$ means a sum over cyclic permutations of $(i j k)$. Its vanishing is explained by noting that it is BRST trivial. For the five-point amplitude (1.1), the generalized BCJ identities of [13, 14] hold in the form of

$$
-\frac{L_{45}}{s_{45}} L_{\{12} V_{3\}}+\frac{L_{42}}{s_{24}} L_{\{13} V_{5\}}-\frac{L_{12}}{s_{12}} L_{\{34} V_{5\}}+\frac{L_{51}}{s_{51}} L_{\{23} V_{4\}}=0,
$$

etc. It is well-known that there are powerful four-dimensional methods to compute scattering amplitudes recursively (see [15] and references therein). The hints of a simplified ten-dimensional parametrization of field theory tree-level amplitudes using pure spinors seem to suggest that there might be similar methods in a ten-dimensional pure spinor superspace setup - which is desirable since there is no need to differentiate between MHV and NMHV contributions as in the four-dimensional methods.

This paper is organized as follows. In section 2 an ansatz will be given for the treelevel five-point SYM amplitude by analogy with the structure of the known four-point amplitude. In section 3 the five-point ansatz will be derived from the field theory limit of a BRST-equivalent expression of the superstring amplitude computed in [12]. In subsection 3.1 an empirical method to write down similar Ansätze for higher-point amplitudes is presented, and expressions for the 6- and 7-point super-Yang-Mills amplitudes in tendimensional space-time are conjectured. In Appendix A the BCJ kinematic relations and its generalization [13,14] are written down using the pure spinor representations of the previous sections. Finally, in Appendix B the first few terms of the (rather long) 5-, 6and 7-gluon expansions from (1.1), (1.2) and (1.3) are written down (the full expansions can be easily generated with a computer using [8] or other methods).

3 It was suggested a long time ago that pure spinors simplify the description of super-Yang-Mills and supergravity theories [16]. The superspace results obtained with the pure spinor formalism seem to realize those expectations. 


\section{Tree-level amplitudes with the pure spinor formalism}

The prescription to compute n-point tree-level open string amplitudes with the pure spinor formalism is given by [2]

$$
\mathcal{A}_{n}=\left\langle V^{1}(0) V^{(n-1)}(1) V^{n}(\infty) \int d z_{2} U^{2}\left(z_{2}\right) \ldots \int d z_{(n-2)} U^{(n-2)}\left(z_{(n-2)}\right\rangle,\right.
$$

where $V^{i}(z)=\lambda^{\alpha} A_{\alpha}^{i}$ and $U^{i}(z)=\partial \theta^{\alpha} A_{\alpha}^{i}+\Pi^{m} A_{m}^{i}+d_{\alpha} W_{i}^{\alpha}+\frac{1}{2} \mathcal{F}_{m n}^{i} N^{m n}$ are the unintegrated and integrated vertices with conformal weight zero and one, respectively, and $i$ is the label denoting the different strings being scattered. The massless sector of the open superstring is described by the ten-dimensionl super-Yang-Mills superfields $\left[A_{\alpha}, A_{m}, W^{\alpha}, \mathcal{F}_{m n}\right]$ which satisfy the equations of motion «1, 19,18,

$$
Q \mathcal{F}_{m n}=2 k_{[m}\left(\lambda \gamma_{n]} W\right), Q W^{\alpha}=\frac{1}{4}\left(\lambda \gamma^{m n}\right)^{\alpha} \mathcal{F}_{m n}, Q A_{m}=\left(\lambda \gamma_{m} W\right)+k_{m}(\lambda A), Q V=0
$$

where $\lambda^{\alpha}(z)$ is a pure spinor satisfying $\lambda^{\alpha} \gamma_{\alpha \beta}^{m} \lambda^{\beta}=0, Q=\lambda^{\alpha} D_{\alpha}$ is the pure spinor BRST operator and $D_{\alpha}=\partial_{\alpha}+\frac{1}{2} k_{m}\left(\gamma^{m} \theta\right)_{\alpha}$ is the supersymmetric derivative 5 . They have the following $\theta$-expansions, [20] 21]

$$
\begin{gathered}
A_{\alpha}(x, \theta)=\frac{1}{2} a_{m}\left(\gamma^{m} \theta\right)_{\alpha}-\frac{1}{3}\left(\xi \gamma_{m} \theta\right)\left(\gamma^{m} \theta\right)_{\alpha}-\frac{1}{32} F_{m n}\left(\gamma_{p} \theta\right)_{\alpha}\left(\theta \gamma^{m n p} \theta\right)+\ldots \\
A_{m}(x, \theta)=a_{m}-\left(\xi \gamma_{m} \theta\right)-\frac{1}{8}\left(\theta \gamma_{m} \gamma^{p q} \theta\right) F_{p q}+\frac{1}{12}\left(\theta \gamma_{m} \gamma^{p q} \theta\right)\left(\partial_{p} \xi \gamma_{q} \theta\right)+\ldots \\
W^{\alpha}(x, \theta)=\xi^{\alpha}-\frac{1}{4}\left(\gamma^{m n} \theta\right)^{\alpha} F_{m n}+\frac{1}{4}\left(\gamma^{m n} \theta\right)^{\alpha}\left(\partial_{m} \xi \gamma_{n} \theta\right)+\frac{1}{48}\left(\gamma^{m n} \theta\right)^{\alpha}\left(\theta \gamma_{n} \gamma^{p q} \theta\right) \partial_{m} F_{p q}+\ldots \\
\mathcal{F}_{m n}(x, \theta)=F_{m n}-2\left(\partial_{[m} \xi \gamma_{n]} \theta\right)+\frac{1}{4}\left(\theta \gamma_{[m} \gamma^{p q} \theta\right) \partial_{n]} F_{p q}+\ldots,
\end{gathered}
$$

where $a_{m}(x)=e_{m} \mathrm{e}^{i k \cdot x}, \xi^{\alpha}(x)=\chi^{\alpha} \mathrm{e}^{i k \cdot x}$ are the bosonic and fermionic polarizations and $F_{m n}=2 \partial_{[m} a_{n]}$ is the field-strength.

After using the OPE's to eliminate the conformal weight-one variables from (2.1), the integration of the zero-modes of $\lambda^{\alpha}$ and $\theta^{\alpha}$ is carried out by taking only the terms which

4 For background material in the pure spinor formalism, see 17, 18. The conventions for the OPE's however follow the appendix A of [12].

5 In what follows spinor index contractions are denoted by parenthesis, e.g. $\lambda^{\alpha} D_{\alpha}=(\lambda D)$ and the worldsheet positions are mostly omitted. 
contain three $\lambda$ 's and five $\theta$ 's in the correlator which are proportional to the pure spinor measure

$$
\left\langle\left(\lambda \gamma^{m} \theta\right)\left(\lambda \gamma^{n} \theta\right)\left(\lambda \gamma^{p} \theta\right)\left(\theta \gamma_{m n p} \theta\right)\right\rangle=1
$$

where the normalization can be chosen arbitrarily6. The normalization condition (2.4) defines the action of the pure spinor angle-brackets \langle\rangle . Arbitrary pure spinor superspace expressions are written down as

$$
\left\langle\lambda^{\alpha} \lambda^{\beta} \lambda^{\gamma} f_{\alpha \beta \gamma}(\theta)\right\rangle
$$

where $f_{\alpha \beta \gamma}(\theta)$ is given in terms of super-Yang-Mills superfields, e.g. $f_{\alpha \beta \gamma}(\theta)=$ $A_{\alpha}^{i}(\theta) A_{\beta}^{j}(\theta) A_{\gamma}^{k}(\theta)$. The measure (2.4) is in the cohomology of the pure spinor BRST operator and can not be written as the supersymmetry variation of a BRST-closed object, so amplitudes computed from (2.1) are supersymmetric [2].

As an illustration of the above steps, the supersymmetric tree-level 3-point amplitude following from (2.1) is given byl

$$
\mathcal{A}_{3}=\left\langle\left(\lambda A^{1}\right)\left(\lambda A^{2}\right)\left(\lambda A^{3}\right)\right\rangle .
$$

Evaluating the explicit component expansion for e.g. the 3-gluon amplitude, is a matter of plugging in the expansions (2.3) and selecting the components with five $\theta$ 's which contain the gluon fields. Doing that one obtains,

$$
\mathcal{A}_{3}=-\frac{1}{64}\left(k_{m}^{3} e_{r}^{1} e_{s}^{2} e_{n}^{3}-k_{m}^{2} e_{r}^{1} e_{n}^{2} e_{s}^{3}+k_{m}^{1} e_{n}^{1} e_{r}^{2} e_{s}^{3}\right)\left\langle\left(\lambda \gamma^{r} \theta\right)\left(\lambda \gamma^{s} \theta\right)\left(\lambda \gamma_{p} \theta\right)\left(\theta \gamma^{p m n} \theta\right)\right\rangle .
$$

As mentioned in the appendix of [23], symmetry arguments and the normalization condition (2.4) fix all pure spinor correlators. Among the list of [23] one finds

$$
\left\langle\left(\lambda \gamma^{r} \theta\right)\left(\lambda \gamma^{s} \theta\right)\left(\lambda \gamma_{p} \theta\right)\left(\theta \gamma^{p m n} \theta\right)\right\rangle=\frac{1}{120} \delta_{p m n}^{r s p}=\frac{1}{45} \delta_{m n}^{r s},
$$

so the 3 -gluon amplitude (2.7) is given by

$$
\mathcal{A}_{3}=-\frac{1}{2880}\left(\left(e^{1} \cdot e^{2}\right)\left(k^{2} \cdot e^{3}\right)+\left(e^{1} \cdot e^{3}\right)\left(k^{1} \cdot e^{2}\right)+\left(e^{2} \cdot e^{3}\right)\left(k^{3} \cdot e^{1}\right)\right) .
$$

Given the systematic nature of the above procedure, an implementation using FORM [24] has been written which performs these expansions automatically [8]. So although component expansions can have many thousand terms as in the 7-gluon amplitude discussed in appendix B, they come from much simpler superspace expressions which can be analysed by hand.

6 See however the tree-level, one-loop and two-loop calculations of [22] to check how the choice has to be taken into account at higher-loops.

7 One also has to evaluate the functional integration of the exponentials $\prod: \mathrm{e}^{i k^{i} \cdot X\left(z_{i}\right)}:$, but they will not appear explicitly in this paper. 


\section{The 5-pt field theory amplitude ansatz}

When the amplitude involves more than three strings, the prescription (2.1) requires the computation of the OPE's with integrated vertices. In this section we will be concerned with the field theory limit (FT) of the string scattering. The 5-point FT amplitude will be given an Ansatz motivated by the superspace form of the FT 4-point amplitude, which will later be obtained from a BRST equivalent expression of the first principles superstring 5 -point amplitude evaluated in [12].

In superspace, the OPE between the unintegrated and integrated vertex operators is given by $V^{i}(z) U^{j}(w) \rightarrow \frac{\tilde{L}_{i j}}{z-w}$, with [25]

$$
\tilde{L}_{i j}(\theta)=A_{m}^{i}\left(\lambda \gamma^{m} W^{j}\right)+\left(\lambda A^{i}\right)\left(k^{i} \cdot A^{j}\right) .
$$

Using the equations of motion (2.2) it follows that

$$
Q \tilde{L}_{i j}=-s_{i j}\left(\lambda A^{i}\right)\left(\lambda A^{j}\right), \quad Q\left(A^{i} \cdot A^{j}\right)=\tilde{L}_{i j}+\tilde{L}_{j i} \equiv 2 \tilde{L}_{(i j)}
$$

wherd $s_{i j}=\left(k^{i} \cdot k^{j}\right)$. Using (3.2) and defining $L_{i j}=1 / 2\left(\tilde{L}_{i j}-\tilde{L}_{j i}\right)$ the superfield $\tilde{L}_{i j}$ can be written as 9

$$
\tilde{L}_{i j}=L_{i j}+\frac{1}{2} Q\left(A^{i} \cdot A^{j}\right) .
$$

The massless 4-point super-Yang-Mills amplitude obtained from the field theory limit of the open string amplitude is given by [12]

$$
\mathcal{A}(1,2,3,4)=\frac{1}{s_{12}}\left\langle\tilde{L}_{12} V^{3} V^{4}\right\rangle+\frac{1}{s_{41}}\left\langle\tilde{L}_{41} V^{2} V^{3}\right\rangle=\frac{1}{s_{12}}\left\langle L_{12} V^{3} V^{4}\right\rangle+\frac{1}{s_{41}}\left\langle L_{41} V^{2} V^{3}\right\rangle
$$

where we used that $\left\langle Q\left(A^{i} \cdot A^{j}\right) V^{k} V^{l}\right\rangle=0$, which follows from integrating the BRST charge by parts. The other sub-amplitudes are obtained from (3.4) by relabeling,

$$
\begin{aligned}
& \mathcal{A}(1,3,4,2)=-\frac{1}{s_{13}}\left\langle L_{13} V^{2} V^{4}\right\rangle-\frac{1}{s_{12}}\left\langle L_{12} V^{3} V^{4}\right\rangle \\
& \mathcal{A}(1,4,2,3)=-\frac{1}{s_{14}}\left\langle L_{41} V^{2} V^{3}\right\rangle+\frac{1}{s_{13}}\left\langle L_{13} V^{2} V^{4}\right\rangle .
\end{aligned}
$$

It is easy to check that the amplitudes in (3.5) are BRST-closed.

8 Note that the usual definition for massless particles is $s_{i j}=2\left(k^{i} \cdot k^{j}\right)$.

9 I thank Dimitrios Tsimpis for suggesting the separation of the BRST-trivial part of $\tilde{L}_{i j}$. 
As emphasized in [5], a color-ordered 5-point tree-level amplitude consists of five diagrams with purely cubic vertices specifying the poles,

$$
\mathcal{A}(1,2,3,4,5)=\frac{n_{1}}{s_{45} s_{12}}+\frac{n_{2}}{s_{51} s_{23}}+\frac{n_{3}}{s_{12} s_{34}}+\frac{n_{4}}{s_{23} s_{45}}+\frac{n_{5}}{s_{34} s_{51}} .
$$

As the BRST variation of $L_{i j}$ is proportional to $s_{i j}$, the idea now is to construct a pure spinor superspace expression using $L_{i j}$ and $L_{k l}$ in the numerators of the terms containing poles in $s_{i j}$ and $s_{k l}$, in such a way as to obtain a BRST-closed expression. It is straightforward to see that the amplitudes

$$
\begin{aligned}
& \mathcal{A}(1,2,3,4,5)=\frac{\left\langle L_{45} L_{12} V^{3}\right\rangle}{s_{45} s_{12}}+\frac{\left\langle L_{51} L_{23} V^{4}\right\rangle}{s_{51} s_{23}}+\frac{\left\langle L_{12} L_{34} V^{5}\right\rangle}{s_{12} s_{34}}+\frac{\left\langle L_{23} L_{45} V^{1}\right\rangle}{s_{23} s_{45}}+\frac{\left\langle L_{34} L_{51} V^{2}\right\rangle}{s_{34} s_{51}} \\
& \mathcal{A}(1,3,2,4,5)=\frac{\left\langle L_{45} L_{13} V^{2}\right\rangle}{s_{45} s_{13}}-\frac{\left\langle L_{51} L_{23} V^{4}\right\rangle}{s_{51} s_{23}}-\frac{\left\langle L_{13} L_{42} V^{5}\right\rangle}{s_{13} s_{24}}-\frac{\left\langle L_{23} L_{45} V^{1}\right\rangle}{s_{23} s_{45}}-\frac{\left\langle L_{42} L_{51} V^{3}\right\rangle}{s_{24} s_{51}} \\
& \mathcal{A}(1,4,3,2,5)=\frac{\left\langle L_{25} L_{14} V^{3}\right\rangle}{s_{25} s_{14}}+\frac{\left\langle L_{34} L_{51} V^{2}\right\rangle}{s_{51} s_{43}}+\frac{\left\langle L_{23} L_{14} V^{5}\right\rangle}{s_{14} s_{32}}+\frac{\left\langle L_{25} L_{34} V^{1}\right\rangle}{s_{43} s_{25}}+\frac{\left\langle L_{51} L_{23} V^{4}\right\rangle}{s_{32} s_{51}} \\
& \mathcal{A}(1,3,4,2,5)=\frac{\left\langle L_{25} L_{13} V^{4}\right\rangle}{s_{25} s_{13}}-\frac{\left\langle L_{34} L_{51} V^{2}\right\rangle}{s_{51} s_{34}}+\frac{\left\langle L_{13} L_{42} V^{5}\right\rangle}{s_{13} s_{42}}-\frac{\left\langle L_{25} L_{34} V^{1}\right\rangle}{s_{34} s_{25}}+\frac{\left\langle L_{42} L_{51} V^{3}\right\rangle}{s_{42} s_{51}} \\
& \mathcal{A}(1,2,4,3,5)=\frac{\left\langle L_{35} L_{12} V^{4}\right\rangle}{s_{35} s_{12}}+\frac{\left\langle L_{42} L_{51} V^{3}\right\rangle}{s_{51} s_{43}}-\frac{\left\langle L_{12} L_{34} V^{5}\right\rangle}{s_{12} s_{43}}+\frac{\left\langle L_{35} L_{42} V^{1}\right\rangle}{s_{42} s_{35}}-\frac{\left\langle L_{34} L_{51} V^{2}\right\rangle}{s_{43} s_{51}} \\
& \mathcal{A}(1,4,2,3,5)=\frac{\left\langle L_{35} L_{14} V^{2}\right\rangle}{s_{35} s_{14}}-\frac{\left\langle L_{42} L_{51} V^{3}\right\rangle}{s_{51} s_{24}}-\frac{\left\langle L_{23} L_{14} V^{5}\right\rangle}{s_{14} s_{23}}-\frac{\left\langle L_{35} L_{42} V^{1}\right\rangle}{s_{24} s_{35}}-\frac{\left\langle L_{51} L_{23} V^{4}\right\rangle}{s_{23} s_{51}}
\end{aligned}
$$

are BRST-closed. One can also check that all sub-amplitudes in (3.7) are related to $A(1,2,3,4,5)$ by index relabeling, taking into account the antisymmetry of $L_{i j}$ and its fermionic nature. The signs in (3.7) precisely match the ones presented in equation (4.5) of [5], so one can identify

$$
\begin{gathered}
n_{1}=\left\langle L_{45} L_{12} V^{3}\right\rangle, n_{2}=\left\langle L_{51} L_{23} V^{4}\right\rangle, n_{3}=\left\langle L_{12} L_{34} V^{5}\right\rangle, n_{4}=\left\langle L_{23} L_{45} V^{1}\right\rangle \\
n_{5}=\left\langle L_{34} L_{51} V^{2}\right\rangle, n_{6}=\left\langle L_{25} L_{14} V^{3}\right\rangle, n_{7}=\left\langle L_{23} L_{14} V^{5}\right\rangle, n_{8}=\left\langle L_{25} L_{34} V^{1}\right\rangle \\
n_{9}=\left\langle L_{25} L_{13} V^{4}\right\rangle, n_{10}=\left\langle L_{13} L_{42} V^{5}\right\rangle, n_{11}=\left\langle L_{42} L_{51} V^{3}\right\rangle, n_{12}=\left\langle L_{35} L_{12} V^{4}\right\rangle \\
n_{13}=\left\langle L_{35} L_{42} V^{1}\right\rangle, \quad n_{14}=\left\langle L_{35} L_{14} V^{2}\right\rangle, \quad n_{15}=\left\langle L_{45} L_{13} V^{2}\right\rangle .
\end{gathered}
$$

As will be mentioned in the appendix, the above "solution" for the $n_{i}$ 's of [5] do not satisfy the strict Bern-Carrasco-Johansson (BCJ) kinematic identities, but they do satisfy the generalized BCJ's of [13,14]. As explained in [13,14], a general parametrization of the sub-amplitudes in terms of poles does not necessarily satisfy the BCJ Jacobi-like identities of [5]. They must however satisfy "generalized BCJ identities", for which the original BCJ relations are just one out of many possible solutions.

The amplitudes in (3.7) will now be obtained from the field theory limit of a BRSTequivalent expression of the pure spinor superstring amplitude computed in [12]. 


\section{First principles derivation of the 5-pt ansatz (3.7)}

The massless 5-point open superstring amplitude is given by [12]

$$
\begin{gathered}
\mathcal{A}_{5}(1,2,3,4,5)=\left\langle L_{2131} V^{4} V^{5}\right\rangle K_{1}-\left\langle L_{2134} V^{5}\right\rangle K_{2}-\left\langle L_{2434} V^{1} V^{5}\right\rangle K_{1}^{\prime}+\left\langle L_{2431} V^{5}\right\rangle K_{3} \\
-\left\langle L_{2331} V^{4} V^{5}\right\rangle K_{5}-\left\langle L_{2334} V^{1} V^{5}\right\rangle K_{4}^{\prime}+\left\langle D_{23} V^{1} V^{4} V^{5}\right\rangle\left(1+s_{23}\right) K_{6}
\end{gathered}
$$

where $K_{j}$ and $K_{j}^{\prime}$ denote integrals which satisfy [26]

$$
\begin{gathered}
s_{34} K_{2}=s_{13} K_{1}+s_{23} K_{4}, \quad s_{24} K_{3}=s_{12} K_{1}-s_{23} K_{5}, \quad K_{1}=K_{4}-K_{5} \\
s_{12} K_{2}=s_{24} K_{1}^{\prime}+s_{23} K_{4}^{\prime}, \quad s_{13} K_{3}=s_{34} K_{1}^{\prime}-s_{23} K_{5}^{\prime}, \quad K_{1}^{\prime}=K_{4}^{\prime}-K_{5}^{\prime} \\
\left(1+s_{23}\right) K_{6}=s_{34} K_{4}^{\prime}-s_{13} K_{5}=s_{12} K_{4}-s_{24} K_{5}^{\prime} .
\end{gathered}
$$

The various $L_{i j k l}$ kinematic building blocks have the following expressions 11

$$
\begin{gathered}
L_{2131}=+\tilde{L}_{12}\left(\left(k^{1}+k^{2}\right) \cdot A^{3}\right)+\left(\lambda \gamma^{m} W^{3}\right)\left[A_{m}^{1}\left(k^{1} \cdot A^{2}\right)+A^{1 n} \mathcal{F}_{m n}^{2}-\left(W^{1} \gamma_{m} W^{2}\right)\right] \\
L_{2134}=\tilde{L}_{12} \tilde{L}_{43}, \quad D_{23}=-\left(A^{2} \cdot A^{3}\right) .
\end{gathered}
$$

Relabeling $1 \leftrightarrow 4$ determines $L_{2434}$ from (4.3) and $L_{2431}$ from (4.4). Finally, the OPE identities of [12] (which are related to the BCJ dualities of [5]) imply that

$$
L_{2331}=L_{3121}-L_{2131}, \quad L_{2334}=L_{3424}-L_{2434},
$$

which are used to obtain the remaining kinematic factors appearing in (4.1) from the expression for (4.3) and relabelings thereof.

Using the integral relation for $K_{6}$ and the expression for $D_{23}$,

$$
\left\langle D_{23} V^{1} V^{4} V^{5}\right\rangle K_{6}=-\left(1+s_{23}\right) K_{6}\left\langle\left(A^{2} \cdot A^{3}\right) V^{1} V^{4} V^{5}\right\rangle=\left(s_{13} K_{5}-s_{34} K_{4}^{\prime}\right)\left\langle\left(A^{2} \cdot A^{3}\right) V^{1} V^{4} V^{5}\right\rangle
$$

the amplitude (4.1) becomes

$$
\mathcal{A}_{5}(1,2,3,4,5)=\left\langle L_{2131} V^{4} V^{5}\right\rangle K_{1}-\left\langle L_{2134} V^{5}\right\rangle K_{2}-\left\langle L_{2434} V^{1} V^{5}\right\rangle K_{1}^{\prime}+\left\langle L_{2431} V^{5}\right\rangle K_{3}
$$

10 The notation here slightly differs from [12], but should not lead to confusion.

11 In the computations of [12] there were terms with factors of $\left(A^{i} W^{j}\right) V^{k}$ in the expressions for $L_{j i k i}$. But it was shown that using the relations (4.2) those terms drop out from the amplitude, so they are not written in this paper for brevity. 


$$
-\left\langle\left(L_{2331}-s_{13}\left(A^{2} \cdot A^{3}\right) V^{1}\right) V^{4} V^{5}\right\rangle K_{5}-\left\langle\left(L_{2334}-s_{34}\left(A^{2} \cdot A^{3}\right) V^{4}\right) V^{1} V^{5}\right\rangle K_{4}^{\prime} .
$$

A key point is to note from (4.3) is that it obeys the identity

$$
Q L_{2131}=s_{12}\left(\tilde{L}_{23} V_{1}-\tilde{L}_{13} V_{1}+\tilde{L}_{12} V_{3}\right)-\left(s_{12}+s_{13}+s_{23}\right) \tilde{L}_{12} V_{3},
$$

and by defining 12

$$
T_{i j k} \equiv L_{j i k i}-S_{j i k i}, \quad S_{j i k i}=\frac{1}{2} s_{i j}\left(\left(A^{j} \cdot A^{k}\right) V^{i}-\left(A^{i} \cdot A^{k}\right) V^{j}\right)-\frac{1}{2}\left(s_{i k}+s_{j k}\right)\left(A^{i} \cdot A^{j}\right) V^{k},
$$

the BRST-trivial parts from $L_{j i k i}$ are removed and one obtains a BRST variation written in terms of $L_{i j}$ instead of $\tilde{L}_{i j}$,

$$
Q T_{i j k}=s_{i j} L_{\{i j} V_{k\}}-\left(s_{j k}+s_{k i}+s_{i j}\right) L_{i j} V_{k} .
$$

Furthermore, using (4.9) it is easy to show that $Q\left(T_{j i k}-T_{j k i}-T_{k i j}\right)=0$. In fact this combination is BRST-trivial,

$$
T_{j i k}-T_{j k i}-T_{k i j}=Q\left(\left(A^{i} \cdot A^{j}\right)\left(k^{i} \cdot A^{k}\right)-\left(A^{i} \cdot A^{k}\right)\left(k^{i} \cdot A^{j}\right)-\left(A^{j} \cdot A^{k}\right)\left(k^{k} \cdot A^{i}\right)\right) .
$$

Using the definitions (3.3), (4.8), the relations (4.2) obeyed by the integrals and the identity (4.10) the superstring five point amplitude (4.6) becomes

$$
\begin{gathered}
\mathcal{A}_{5}(1,2,3,4,5)=\left\langle L_{12} L_{34} V_{5}\right\rangle K_{2}+\left\langle L_{13} L_{24} V_{5}\right\rangle K_{3} \\
+\left\langle T_{123} V_{4} V_{5}\right\rangle K_{1}-\left\langle T_{432} V_{1} V_{5}\right\rangle K_{1}^{\prime}+\left\langle T_{321} V_{4} V_{5}\right\rangle K_{5}-\left\langle T_{234} V_{1} V_{5}\right\rangle K_{5}^{\prime} .
\end{gathered}
$$

As discussed in [26], under the twist $2 \leftrightarrow 3$ and $1 \leftrightarrow 4$ of the vertex operators on the disc, the integrals behave as

$$
K_{1} \leftrightarrow K_{1}^{\prime}, \quad K_{4} \leftrightarrow K_{4}^{\prime}, \quad K_{5} \leftrightarrow K_{5}^{\prime}, \quad K_{2} \leftrightarrow K_{2}, \quad K_{3} \leftrightarrow K_{3},
$$

from which one can easily check that the 5-pt superstring amplitude (4.11) is antisymmetric, as it should on general grounds.

Writing the five point integrals in the two dimensional basis $\left(T, K_{3}\right)$ of [26] where

$$
T=s_{12} s_{34} K_{2}+\left(s_{12} s_{51}-s_{12} s_{34}+s_{34} s_{45}\right) K_{3}
$$

12 I thank Dimitrios Tsimpis for suggesting the relevance of using this definition in the context of an ansatz for the 6-pt amplitude. It turns out to clean up the 5-pt formulæ too. 
as follows 12

$$
\begin{gathered}
K_{1}=\frac{T}{s_{12} s_{45}}-\left(\frac{s_{34}}{s_{12}}+\frac{s_{23}}{s_{45}}\right) K_{3}, \quad K_{1}^{\prime}=\frac{T}{s_{34} s_{51}}-\left(\frac{s_{12}}{s_{34}}+\frac{s_{23}}{s_{51}}\right) K_{3} \\
K_{5}=\frac{T}{s_{23} s_{45}}-\left(\frac{s_{12}}{s_{45}}+\frac{s_{51}}{s_{23}}-1\right) K_{3}, \quad K_{5}^{\prime}=\frac{T}{s_{23} s_{51}}-\left(\frac{s_{34}}{s_{51}}+\frac{s_{45}}{s_{23}}-1\right) K_{3}
\end{gathered}
$$

the amplitude (4.11) becomes

$$
\mathcal{A}_{5}(1,2,3,4,5)=T A_{\mathrm{YM}}(\theta)+K_{3} A_{F^{4}}(\theta)
$$

where,

$$
A_{\mathrm{YM}}(\theta)=\frac{\left\langle T_{123} V^{4} V^{5}\right\rangle}{s_{12} s_{45}}-\frac{\left\langle T_{234} V^{1} V^{5}\right\rangle}{s_{23} s_{51}}+\frac{\left\langle L_{12} L_{34} V^{5}\right\rangle}{s_{12} s_{34}}+\frac{\left\langle T_{321} V^{4} V^{5}\right\rangle}{s_{23} s_{45}}-\frac{\left\langle T_{432} V^{1} V^{5}\right\rangle}{s_{34} s_{51}}
$$

and

$$
\begin{gathered}
A_{F^{4}}(\theta)=\left\langle L_{12} L_{34} V^{5}\right\rangle+\left\langle L_{13} L_{24} V^{5}\right\rangle-\left\langle T_{234} V^{1} V^{5}\right\rangle+\left\langle T_{321} V^{4} V^{5}\right\rangle \\
-\left\langle L_{12} L_{34} V^{5}\right\rangle\left(\frac{s_{45}}{s_{12}}+\frac{s_{51}}{s_{34}}\right)-\left\langle T_{123} V^{4} V^{5}\right\rangle\left(\frac{s_{34}}{s_{12}}+\frac{s_{23}}{s_{45}}\right)+\left\langle T_{234} V^{1} V^{5}\right\rangle\left(\frac{s_{45}}{s_{23}}+\frac{s_{34}}{s_{51}}\right) \\
-\left\langle T_{321} V^{4} V^{5}\right\rangle\left(\frac{s_{51}}{s_{23}}+\frac{s_{12}}{s_{45}}\right)+\left\langle T_{432} V^{1} V^{5}\right\rangle\left(\frac{s_{23}}{s_{51}}+\frac{s_{12}}{s_{34}}\right) .
\end{gathered}
$$

One can also find a BRST-equivalent form for the amplitude by using the fact that $Q\left(L_{m n} / s_{m n}\right)=-V^{m} V^{n}$ to rewrite $\left\langle T_{i j k} V^{m} V^{n}\right\rangle$ as $-\left\langle T_{i j k} Q\left(L_{m n} / s_{m n}\right)\right\rangle$, which upon integration of the BRST charge by parts using (4.9) implies that

$$
\left\langle T_{i j k} V_{m} V_{m}\right\rangle=-\left\langle\frac{L_{m n}}{s_{m n}}\left(s_{i j} L_{\{i j} V_{k\}}-s_{i j k} L_{i j} V_{k}\right)\right\rangle .
$$

A somewhat tedious but straightforward use of (4.19) in the expressions (4.17) and (4.18) allows them to be rewritten as

$$
A_{\mathrm{YM}}(\theta)=\frac{\left\langle L_{45} L_{12} V^{3}\right\rangle}{s_{45} s_{12}}+\frac{\left\langle L_{51} L_{23} V^{4}\right\rangle}{s_{51} s_{23}}+\frac{\left\langle L_{12} L_{34} V^{5}\right\rangle}{s_{12} s_{34}}+\frac{\left\langle L_{23} L_{45} V^{1}\right\rangle}{s_{23} s_{45}}+\frac{\left\langle L_{34} L_{51} V^{2}\right\rangle}{s_{34} s_{51}}
$$

and

$$
\begin{gathered}
A_{F^{4}}(\theta)=-\left\langle L_{45} L_{12} V^{3}\right\rangle\left(\frac{s_{23}}{s_{45}}+\frac{s_{34}}{s_{12}}\right)-\left\langle L_{51} L_{23} V^{4}\right\rangle\left(\frac{s_{34}}{s_{15}}+\frac{s_{45}}{s_{23}}\right) \\
-\left\langle L_{12} L_{34} V^{5}\right\rangle\left(\frac{s_{45}}{s_{12}}+\frac{s_{51}}{s_{34}}\right)-\left\langle L_{23} L_{45} V^{1}\right\rangle\left(\frac{s_{51}}{s_{23}}+\frac{s_{12}}{s_{45}}\right)-\left\langle L_{34} L_{51} V^{2}\right\rangle\left(\frac{s_{12}}{s_{34}}+\frac{s_{23}}{s_{51}}\right) \\
+\left\langle L_{12} L_{34} V^{5}+L_{51} L_{23} V^{4}-L_{13} L_{42} V^{5}+L_{23} L_{45} V^{1}\right\rangle+\frac{s_{13}}{s_{51}}\left\langle L_{51} L_{\{23} V_{4\}}\right\rangle-\frac{s_{24}}{s_{45}}\left\langle L_{45} L_{\{12} V_{3\}}\right\rangle .
\end{gathered}
$$

In the field theory limit $T \rightarrow 1$ and $K_{3} \rightarrow 0$ [26], so the first principles derivation of (3.7) is completed. The 5-gluon component expansion were already computed in [12], and shown to agree with earlier RNS results [26]. 


\subsection{Higher-point amplitudes}

It is worth checking whether the simple mappings between the cubic Feynman diagrams and pure spinor building blocks persist at higher-points. The discussion in section 2 suggests a way to write down n-point field theory amplitudes. For each one of the $2^{n-2}(2 n-5) ! ! /(n-1)$ ! color-ordered diagrams specifying the kinematic poles [5], a ghostnumber-three numerator whose BRST transformation is proportional to those poles should be written down. One then tries to find a combination with the correct dimension of a n-point amplitude such that the sum of all diagrams is BRST-closed.

To help finding candidates for superfield building blocks, the first principles tree-level superstring amplitude prescription [2.27] can be used as guide. For example, the superfield $\tilde{L}_{i j}$ appears in the OPE of $V^{i}(z) U^{j}(w)$ in the 4-pt string amplitude [25, and its BRST transformation $Q \tilde{L}_{i j}=-s_{i j} V^{i} V^{j}$ has precisely the Mandelstam variable to cancel poles in the 5-pt amplitude. Similarly, the superfield $L_{j i k i}$ comes from the numerator of the $1 / z_{i j} z_{i k}$ pole in the OPE $V^{i}\left(z_{i}\right) U^{j}\left(z_{j}\right) U^{k}\left(z_{k}\right)$ appearing in the 5-pt computation [12], and its BRST transformation has the required Mandelstam variables to cancel poles in the 6-pt amplitude,

$$
Q L_{j i k i}=s_{i j}\left(\tilde{L}_{j k} V^{i}-\tilde{L}_{i k} V^{j}+\tilde{L}_{i j} V^{k}\right)-\left(s_{j k}+s_{k i}+s_{i j}\right) \tilde{L}_{i j} V^{k}
$$

As the expressions must be in the cohomology of the pure spinor BRST operator, one also removes the BRST-trivial parts of the building blocks $\tilde{L}_{i j}$ and $L_{j i k i}$, using $L_{i j}$ and $T_{i j k}$ instead.

Following the above procedure for the 14 color-ordered diagrams of the 6-point amplitude which are generated from the cyclic permutations of the diagrams in Figures 1, 2 and 3, a BRST-closed expression with the correct pole structure looks like 13

$$
\begin{gathered}
\mathcal{A}_{6}(1,2,3,4,5,6)=\frac{\left\langle L_{12} L_{34} L_{56}\right\rangle}{3 s_{1} s_{3} s_{5}} \\
+\frac{1}{2} \frac{\left\langle T_{123}\right.}{s_{1} t_{1}} \frac{\left(V^{4} L_{56}\right.}{s_{5}}+\frac{\left.\left.L_{45} V^{6}\right)\right\rangle}{s_{4}}-\frac{1}{2} \frac{\left\langle T_{126}\right.}{s_{1} t_{3}} \frac{\left(V^{3} L_{45}\right.}{s_{4}}+\frac{\left.\left.L_{34} V^{5}\right)\right\rangle}{s_{3}}+\operatorname{cyclic}(1 \ldots 6)
\end{gathered}
$$

where $s_{1}=s_{12}, s_{2}=s_{23}, \ldots, s_{6}=s_{61}, t_{1}=\left(s_{12}+s_{23}+s_{13}\right), t_{2}=\left(s_{23}+s_{34}+s_{24}\right)$ and $t_{3}=\left(s_{34}+s_{45}+s_{35}\right)$ are the 6-point Mandelstam variables of [6]. The full component expansion for the 6-gluon amplitude obtained from (4.23) contains 6706 terms [8] and it

13 I thank Oliver Schlotterer and Dimitrios Tsimpis for many valuable discussions. 
was checked to be gauge invariant 14 . The first few terms of this expansion are given in Appendix B.

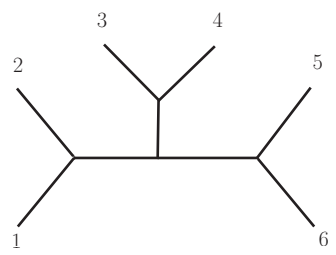

Fig. 1. The diagram associated with $\left\langle\frac{L_{12}}{s_{1}} \frac{L_{34}}{s_{3}} \frac{L_{56}}{s_{5}}\right\rangle$.

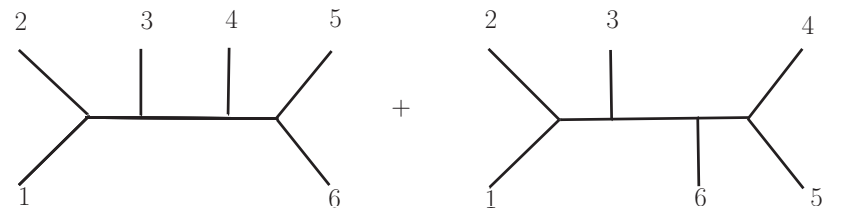

Fig. 2. The diagrams associated with $\left\langle\frac{T_{123}}{s_{1} t_{1}}\left(\frac{V_{4} L_{56}}{s_{5}}+\frac{L_{45} V_{6}}{s_{4}}\right)\right\rangle$.

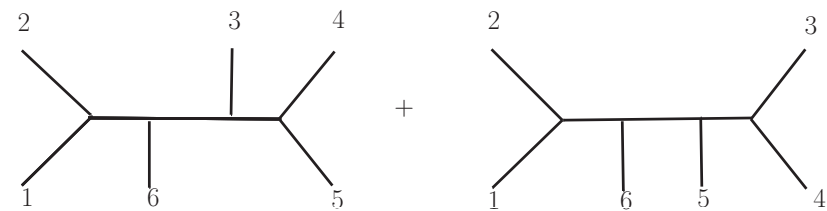

Fig. 3. The diagrams associated with $\left\langle\frac{T_{126}}{s_{1} t_{3}}\left(\frac{V_{3} L_{45}}{s_{4}}+\frac{L_{34} V_{5}}{s_{3}}\right)\right\rangle$.

For the 7-point amplitude there are 6 diagrams which generate the 42 color-ordered cubic diagrams upon cyclic symmetrization. The corresponding BRST-closed expression with the correct pole structure is given by

$$
\begin{array}{r}
\mathcal{A}_{7}(1,2,3,4,5,6,7)=+\frac{\left\langle T_{231} L_{45} L_{67}\right\rangle}{s_{2} t_{1} s_{4} s_{6}}+\frac{\left\langle T_{123} T_{564} V_{7}\right\rangle}{s_{1} t_{1} s_{5} t_{4}}+\frac{\left\langle T_{127} T_{345} V_{6}\right\rangle}{s_{1} t_{7} s_{3} t_{3}} \\
-\frac{\left\langle T_{123} T_{456} V_{7}\right\rangle}{s_{1} t_{1} s_{4} t_{4}}-\frac{\left\langle T_{127} T_{453} V_{6}\right\rangle}{s_{1} t_{7} s_{4} t_{3}}-\frac{\left\langle T_{123} L_{45} L_{67}\right\rangle}{s_{1} t_{1} s_{4} s_{6}}+\operatorname{cyclic}(1 \ldots 7)
\end{array}
$$

where $s_{1}, \ldots, s_{7}$ and $t_{1}, \ldots, t_{7}$ are the 7-point Mandelstam variables of [7]. The tendimensional 7-gluon expansion of (4.24) contains more than 130 thousand terms [8] and a few are written in appendix B. As the results of [7] are written in the four-dimensional helicity formalism, a direct comparison with the results quoted there is not possible.

14 After the first version of this paper appeared, Zvi Bern kindly provided his Mathematica file with the field theory 6-gluon amplitude written in terms of polarization and momenta. A perfect match was obtained. 
Acknowledgements: I want to thank Nathan Berkovits, Oliver Schlotterer, Stephan Stieberger and Dimitrios Tsimpis for discussions and for comments on the draft. I also thank the organizers of the Amsterdam String Theory Workshop 2010 for the inspiring atmosphere, as the final details of this paper were worked out during those days. I thank the Werner-Heisenberg-Institut in München for hospitality and partial financial support, and in particular Stephan Stieberger and Oliver Schlotterer for their invitations and warm hospitality. I acknowledge support by the Deutsch-Israelische Projektkooperation (DIP H52).

\section{Appendix A. The Bern-Carrasco-Johansson kinematic identities}

The 4-pt BCJ kinematic relation $n_{u}=n_{s}-n_{t}$ is mapped to the superspace expression $\left\langle L_{13} V^{2} V^{4}\right\rangle=\left\langle L_{12} V^{3} V^{4}\right\rangle-\left\langle L_{41} V^{2} V^{3}\right\rangle$. Using $\left\langle L_{41} V^{2} V^{3}\right\rangle=-\left\langle L_{23} V^{1} V^{4}\right\rangle$ it can be rewritten as

$$
\left\langle L_{\{12} V_{3\}} V^{4}\right\rangle=0,
$$

where $\{i j k\}$ means to sum over the cyclic permutation of the labels. Note that (A.1) can be explained from the fact that BRST-trivial quantities vanish. Explicitly,

$$
0=\left\langle Q\left(T_{123} V_{4}\right)\right\rangle=s\left\langle L_{\{12} V_{3\}} V^{4}\right\rangle-(s+t+u)\left\langle L_{12} V_{3} V_{4}\right\rangle
$$

which implies (A.1) because $s+t+u=0$.

The 5-pt extended BCJ relations of [13] 14] are given by

$$
\begin{aligned}
& \frac{n_{4}-n_{1}+n_{15}}{s_{45}}-\frac{n_{10}-n_{11}+n_{13}}{s_{24}}-\frac{n_{3}-n_{1}+n_{12}}{s_{12}}-\frac{n_{5}-n_{2}+n_{11}}{s_{51}}=0 \\
& \frac{n_{7}-n_{6}+n_{14}}{s_{14}}-\frac{n_{10}-n_{11}+n_{13}}{s_{24}}-\frac{n_{8}-n_{6}+n_{9}}{s_{25}}-\frac{n_{5}-n_{2}+n_{11}}{s_{51}}=0 \\
& \frac{n_{10}-n_{9}+n_{15}}{s_{13}}+\frac{n_{5}-n_{2}+n_{11}}{s_{51}}-\frac{n_{4}-n_{2}+n_{7}}{s_{23}}+\frac{n_{8}-n_{6}+n_{9}}{s_{25}}=0 \\
& \frac{n_{4}-n_{1}+n_{15}}{s_{45}}-\frac{n_{10}-n_{9}+n_{15}}{s_{13}}-\frac{n_{5}-n_{2}+n_{11}}{s_{51}}-\frac{n_{3}-n_{5}+n_{8}}{s_{34}}=0 .
\end{aligned}
$$

Using the mappings of (3.8) they become

$$
\begin{aligned}
& -\frac{L_{45}}{s_{45}} L_{\{12} V_{3\}}+\frac{L_{42}}{s_{24}} L_{\{13} V_{5\}}-\frac{L_{12}}{s_{12}} L_{\{34} V_{5\}}+\frac{L_{51}}{s_{51}} L_{\{23} V_{4\}}=0, \\
& -\frac{L_{14}}{s_{14}} L_{\{23} V_{5\}}+\frac{L_{42}}{s_{24}} L_{\{13} V_{5\}}-\frac{L_{25}}{s_{25}} L_{\{13} V_{4\}}+\frac{L_{51}}{s_{51}} L_{\{23} V_{4\}}=0,
\end{aligned}
$$




$$
\begin{aligned}
& +\frac{L_{13}}{s_{13}} L_{\{25} V_{4\}}-\frac{L_{51}}{s_{51}} L_{\{23} V_{4\}}-\frac{L_{23}}{s_{23}} L_{\{14} V_{5\}}+\frac{L_{25}}{s_{25}} L_{\{13} V_{4\}}=0, \\
& -\frac{L_{45}}{s_{45}} L_{\{12} V_{3\}}-\frac{L_{13}}{s_{13}} L_{\{25} V_{4\}}+\frac{L_{51}}{s_{51}} L_{\{23} V_{4\}}+\frac{L_{34}}{s_{34}} L_{\{12} V_{5\}}=0
\end{aligned}
$$

which one can check to hold true when expanding in components. Using the momentum conservation relations

$$
\begin{gathered}
s_{13}=s_{45}-s_{12}-s_{23}, \quad s_{14}=s_{23}-s_{51}-s_{45}, \quad s_{24}=s_{51}-s_{23}-s_{34} \\
s_{25}=s_{34}-s_{12}-s_{51}, \quad s_{35}=s_{12}-s_{45}-s_{34},
\end{gathered}
$$

one finds that the LHS of (A.7) - (A.10) are BRST-closed.

\section{Appendix B. The 5-, 6- and 7-gluon amplitudes}

The 5-gluon amplitude is easily obtained by using [8], and one can check that the first few terms are

$$
\begin{gathered}
2880 \mathcal{A}_{5}(1,2,3,4,5)= \\
-\left(k^{1} \cdot e^{2}\right)\left(k^{1} \cdot e^{3}\right)\left(k^{1} \cdot e^{4}\right)\left(e^{1} \cdot e^{5}\right) s_{1}^{-1} s_{4}^{-1}+\left(k^{1} \cdot e^{2}\right)\left(k^{1} \cdot e^{3}\right)\left(k^{1} \cdot e^{5}\right)\left(e^{1} \cdot e^{4}\right) s_{1}^{-1} s_{4}^{-1} \\
-\left(k^{1} \cdot e^{2}\right)\left(k^{1} \cdot e^{3}\right)\left(k^{2} \cdot e^{4}\right)\left(e^{1} \cdot e^{5}\right) s_{1}^{-1} s_{4}^{-1}+\left(k^{1} \cdot e^{2}\right)\left(k^{1} \cdot e^{3}\right)\left(k^{2} \cdot e^{5}\right)\left(e^{1} \cdot e^{4}\right) s_{1}^{-1} s_{4}^{-1} \\
-\left(k^{1} \cdot e^{2}\right)\left(k^{1} \cdot e^{3}\right)\left(k^{3} \cdot e^{4}\right)\left(e^{1} \cdot e^{5}\right) s_{1}^{-1} s_{3}^{-1}+\ldots
\end{gathered}
$$

The 6-gluon component expansion from the ansatz (4.23) generates 6706 terms of which the first few are [8]

$$
\begin{gathered}
2880 \mathcal{A}_{6}(1,2,3,4,5,6)= \\
{\left[\left(k^{1} \cdot e^{2}\right)\left(k^{1} \cdot e^{3}\right)\left(k^{1} \cdot e^{4}\right)\left(k^{1} \cdot e^{6}\right)\left(e^{1} \cdot e^{5}\right)-\left(k^{1} \cdot e^{2}\right)\left(k^{1} \cdot e^{3}\right)\left(k^{1} \cdot e^{4}\right)\left(k^{1} \cdot e^{5}\right)\left(e^{1} \cdot e^{6}\right)\right.} \\
-\left(k^{1} \cdot e^{2}\right)\left(k^{1} \cdot e^{3}\right)\left(k^{1} \cdot e^{4}\right)\left(k^{2} \cdot e^{5}\right)\left(e^{1} \cdot e^{6}\right)+\left(k^{1} \cdot e^{2}\right)\left(k^{1} \cdot e^{3}\right)\left(k^{1} \cdot e^{4}\right)\left(k^{2} \cdot e^{6}\right)\left(e^{1} \cdot e^{5}\right) \\
\left.-\left(k^{1} \cdot e^{2}\right)\left(k^{1} \cdot e^{3}\right)\left(k^{1} \cdot e^{4}\right)\left(k^{3} \cdot e^{5}\right)\left(e^{1} \cdot e^{6}\right)+\left(k^{1} \cdot e^{2}\right)\left(k^{1} \cdot e^{3}\right)\left(k^{1} \cdot e^{4}\right)\left(k^{3} \cdot e^{6}\right)\left(e^{1} \cdot e^{5}\right)\right] s_{1}^{-1} s_{5}^{-1} t_{1}^{-1} \\
-\left(k^{1} \cdot e^{2}\right)\left(k^{1} \cdot e^{3}\right)\left(k^{1} \cdot e^{4}\right)\left(k^{4} \cdot e^{5}\right)\left(e^{1} \cdot e^{6}\right) s_{1}^{-1} s_{4}^{-1} t_{1}^{-1}+\ldots
\end{gathered}
$$

Similarly, the 7-gluon component expansion of (4.24) has 134460 terms 15 and the first ones are

$$
2880 \mathcal{A}_{7}(1,2,3,4,5,6,7)=
$$

15 Some of those terms contain $\epsilon_{10}$ tensors and are expected to vanish once rules for the vanishing of things like $\epsilon_{10}^{\left[m_{1} \ldots m_{10}\right.} \delta_{n}^{\left.m_{11}\right]}$ are implemented in [8]. 


$$
\begin{gathered}
{\left[+\left(k^{1} \cdot e^{2}\right)\left(k^{1} \cdot e^{3}\right)\left(k^{1} \cdot e^{4}\right)\left(k^{1} \cdot e^{5}\right)\left(k^{1} \cdot e^{6}\right)\left(e^{1} \cdot e^{7}\right)-\left(k^{1} \cdot e^{2}\right)\left(k^{1} \cdot e^{3}\right)\left(k^{1} \cdot e^{4}\right)\left(k^{1} \cdot e^{5}\right)\left(k^{1} \cdot e^{7}\right)\left(e^{1} \cdot e^{6}\right)\right.} \\
+\left(k^{1} \cdot e^{2}\right)\left(k^{1} \cdot e^{3}\right)\left(k^{1} \cdot e^{4}\right)\left(k^{1} \cdot e^{5}\right)\left(k^{2} \cdot e^{6}\right)\left(e^{1} \cdot e^{7}\right)-\left(k^{1} \cdot e^{2}\right)\left(k^{1} \cdot e^{3}\right)\left(k^{1} \cdot e^{4}\right)\left(k^{1} \cdot e^{5}\right)\left(k^{2} \cdot e^{7}\right)\left(e^{1} \cdot e^{6}\right) \\
\left.+\left(k^{1} \cdot e^{2}\right)\left(k^{1} \cdot e^{3}\right)\left(k^{1} \cdot e^{4}\right)\left(k^{1} \cdot e^{5}\right)\left(k^{3} \cdot e^{6}\right)\left(e^{1} \cdot e^{7}\right)\right] s_{1}^{-1} s_{6}^{-1} t_{1}^{-1} t_{5}^{-1}+\ldots
\end{gathered}
$$

It is curious to note that the coefficient of $\pm 1 / 2880$ is the same for all the terms in the 5 -, 6- and 7-gluon amplitudes alike. This is the same coefficient which was observed in [27] to be the conversion factor required to match the RNS amplitudes at tree-level.

\section{Appendix C. Shortcut to compute QL}

There is a shortcut to compute $Q L$ 's for $n$-points using only the $L$ 's appearing at $(n-1)$-points. The definitions of $\tilde{L}_{i j}$ and $L_{j i k i}$ are [12],

$$
V^{i}\left(z_{i}\right) U^{j}\left(z_{j}\right) \rightarrow \frac{\tilde{L}_{i j}}{z_{i j}}, \quad \tilde{L}_{i j}\left(z_{i}\right) U^{k}\left(z_{k}\right) \rightarrow \frac{L_{j i k i}}{z_{i k}}
$$

so that $Q \tilde{L}_{i j}=\lim _{z_{j} \rightarrow z_{i}} z_{i j} Q\left(V^{i}\left(z_{i}\right) U^{j}\left(z_{j}\right)\right)$ and $Q L_{j i k i}=\lim _{z_{k} \rightarrow z_{i}} z_{i k} Q\left(\tilde{L}_{i j}\left(z_{i}\right) U^{k}\left(z_{k}\right)\right)$ leads to

$$
\begin{gathered}
Q \tilde{L}_{i j}=\lim _{z_{j} \rightarrow z_{i}} z_{i j} \partial V^{j}\left(z_{j}\right) V^{i}\left(z_{i}\right)=-s_{i j} V^{i} V^{j} \\
Q L_{j i k i}=-\lim _{z_{k} \rightarrow z_{i}} z_{i k}\left(s_{i j} V^{i}\left(z_{i}\right) V^{j}\left(z_{i}\right) U^{k}\left(z_{k}\right)+\tilde{L}_{i j}\left(z_{i}\right) \partial V^{k}\left(z_{k}\right)\right) \\
=-s_{i j}\left(\tilde{L}_{i k}\left(z_{i}\right) V^{j}\left(z_{i}\right)+V^{i}\left(z_{i}\right) \tilde{L}_{j k}\left(z_{i}\right)\right)+\left(s_{i k}+s_{j k}\right) V^{k}\left(z_{i}\right) \tilde{L}_{i j}\left(z_{i}\right)
\end{gathered}
$$

which agree with (3.2) and (4.22), respectively. In the above we used $Q U^{i}(z)=\partial V^{i}(z)=$ $\Pi^{m}(z) k_{m}^{i} V^{i}(z)+\partial \theta^{\alpha} D_{\alpha} V^{i}(z)+\partial \lambda^{\alpha} A_{\alpha}^{i}$, which together with the OPE's of the conformal weight-one variables [28, 17] implies that

$$
\lim _{z_{i} \rightarrow z_{j}} Q\left(U^{i}\left(z_{i}\right) V^{j}\left(z_{j}\right)\right)=\lim _{z_{i} \rightarrow z_{j}} \partial V^{i}\left(z_{i}\right) V^{j}\left(z_{j}\right) \rightarrow-s_{i j} \frac{V^{i}\left(z_{i}\right) V^{j}\left(z_{i}\right)}{z_{i j}}
$$




\section{References}

[1] S. J. Parke and T. R. Taylor, "An Amplitude for $n$ Gluon Scattering," Phys. Rev. Lett. 56, 2459 (1986).

[2] N. Berkovits, "Super-Poincare covariant quantization of the superstring," JHEP 0004, 018 (2000) arXiv:hep-th/0001035.

[3] N. Berkovits, "Explaining pure spinor superspace," arXiv:hep-th/0612021.

[4] E. Witten, "Twistor - Like Transform In Ten-Dimensions," Nucl. Phys. B 266, 245 (1986).

[5] Z. Bern, J. J. M. Carrasco and H. Johansson, "New Relations for Gauge-Theory Amplitudes," Phys. Rev. D 78, 085011 (2008) arXiv:0805.3993 [hep-ph]].

[6] S. Stieberger and T. R. Taylor, "Multi-gluon scattering in open superstring theory," Phys. Rev. D 74, 126007 (2006) arXiv:hep-th/0609175.

[7] S. Stieberger and T. R. Taylor, "Supersymmetry Relations and MHV Amplitudes in Superstring Theory," Nucl. Phys. B 793, 83 (2008) arXiv:0708.0574 [hep-th]].

[8] C. R. Mafra, "PSS: A FORM Program to Evaluate Pure Spinor Superspace Expressions," arXiv:1007.4999 [hep-th].

[9] D. Oprisa and S. Stieberger, "Six gluon open superstring disk amplitude, multiple hypergeometric series and Euler-Zagier sums," arXiv:hep-th/0509042.

[10] C. Mafra, O. Schlotterer, S. Stieberger, D. Tsimpis, work in progress

[11] Zvi Bern, private communication.

[12] C. R. Mafra, "Simplifying the Tree-level Superstring Massless Five-point Amplitude," JHEP 1001, 007 (2010) [arXiv:0909.5206 [hep-th]].

[13] H. Tye and Y. Zhang, "Dual Identities inside the Gluon and the Graviton Scattering Amplitudes," arXiv:1003.1732 [hep-th].

[14] N. E. J. Bjerrum-Bohr, P. H. Damgaard, T. Sondergaard and P. Vanhove, "Monodromy and Jacobi-like Relations for Color-Ordered Amplitudes," arXiv:1003.2403 [hep-th].

[15] J. M. Drummond and J. M. Henn, "All tree-level amplitudes in N=4 SYM," JHEP 0904, 018 (2009) [arXiv:0808.2475 [hep-th]].

[16] P. S. Howe, "Pure Spinors Lines In Superspace And Ten-Dimensional Supersymmetric Theories," Phys. Lett. B 258, 141 (1991) [Addendum-ibid. B 259, 511 (1991)]. ;

P. S. Howe, "Pure Spinors, Function Superspaces And Supergravity Theories In TenDimensions And Eleven-Dimensions," Phys. Lett. B 273, 90 (1991).

[17] N. Berkovits, "ICTP lectures on covariant quantization of the superstring," arXiv:hepth/0209059.

[18] C. R. Mafra, "Superstring Scattering Amplitudes with the Pure Spinor Formalism," arXiv:0902.1552 [hep-th]. 
[19] N. Berkovits, "Multiloop amplitudes and vanishing theorems using the pure spinor formalism for the superstring," JHEP 0409, 047 (2004) [arXiv:hep-th/0406055].

[20] J. P. Harnad and S. Shnider, "Constraints And Field Equations For Ten-Dimensional Superyang-Mills Theory," Commun. Math. Phys. 106, 183 (1986). ;

H. Ooguri, J. Rahmfeld, H. Robins and J. Tannenhauser, "Holography in superspace," JHEP 0007, 045 (2000) arXiv:hep-th/0007104]. ;

P. A. Grassi and L. Tamassia, "Vertex operators for closed superstrings," JHEP 0407, 071 (2004) arXiv:hep-th/0405072.

[21] G. Policastro and D. Tsimpis, "R**4, purified," Class. Quant. Grav. 23, 4753 (2006) arXiv:hep-th/0603165.

[22] H. Gomez and C. R. Mafra, "The Overall Coefficient of the Two-loop Superstring Amplitude Using Pure Spinors," JHEP 1005, 017 (2010) [arXiv:1003.0678 [hep-th]].

[23] N. Berkovits and C. R. Mafra, "Some superstring amplitude computations with the non-minimal pure spinor formalism," JHEP 0611, 079 (2006) [arXiv:hep-th/0607187].

[24] J. A. M. Vermaseren, "New features of FORM," arXiv:math-ph/0010025. ;

M. Tentyukov and J. A. M. Vermaseren, "The multithreaded version of FORM," arXiv:hep-ph/0702279.

[25] C. R. Mafra, "Pure Spinor Superspace Identities for Massless Four-point Kinematic Factors," JHEP 0804, 093 (2008) [arXiv:0801.0580 [hep-th]].

[26] R. Medina, F. T. Brandt and F. R. Machado, "The open superstring 5-point amplitude revisited," JHEP 0207, 071 (2002) arXiv:hep-th/0208121]. ;

L. A. Barreiro and R. Medina, "5-field terms in the open superstring effective action," JHEP 0503, 055 (2005) arXiv:hep-th/0503182.

[27] N. Berkovits and B. C. Vallilo, "Consistency of super-Poincare covariant superstring tree amplitudes," JHEP 0007, 015 (2000) [arXiv:hep-th/0004171].

[28] W. Siegel, "Classical Superstring Mechanics," Nucl. Phys. B 263, 93 (1986). 